\title{
Zur Frage der Wirkung von Insulin auf die intestinale Resorption von Glucose und Fructose*
}

\author{
Hellmot Mehnert, Harald Förster und Gertrude Haslbeck
}

Medizinische Poliklinik der Universität München (Direktor : Professor Dr. med. W. Seitz)

Eingegangen am 20. Dezember 1965

\begin{abstract}
Investigations concerning the insulin effect on intestinal resorption of glucose and fructose.

Summary. The effect of insulin and of insulin-induced hypoglycemia on the absorption of glucose and fructose solutions was investigated in 282 rats. After administration of these monosaccharides by stomach tube an accelerated absorption of g]ucose, but not of fructose, was found under the influence of insulin. It became evident, though, that glucose absorption was faster only when larger amounte of glucose per unit time were offered to the intestine. $\mathrm{By}$ further investigations the hypothesis could be proven that accelerated glucose absorption after insulin application was due to faster emptying of the stomach. If glucose and fructose were perfused through the intestine, by $=$ passing the stomach, no differences between insulin $=$ treated animals and controls were found. In the light of these findings, the assumption is not longer tenable that a low blood glucose level leads to an increase in the concentration difference between gut and blood and thereby to faster glucose absorption. After i.v. insulin application, 10 minutes before the perfusion of the small intestine had been started, there was even a statistically significant inhibition of glucose absorption. It seems likely, however, that this inhibition of absorption is due to the hypoglycemic condition of the animals. Pretreatment with insulin for one week also did not lead to accelerated glucose resorption.
\end{abstract}

Observations concernant le rôle de l'insuline dans la résorption intestinale du glucose et du fructose.

Résumé. L'effet de l'insuline et de l'hypoglycémie par l'insuline sur la résorption de solutions de glucose et de fructose a été étudié chez 282 rats. Après administration de ces monosaccharides par sonde gastrique, nous avons noté une résorption accélérée du glucose, mais pas du fructose, sous l'influence de l'insuline. Cependant nous avons été frappés par le fait que la résorption accélérée du glucose était liée à des quantités plus élevées de glucose offertes à l'intestin par unité de temps. Au cours de nos recherches, la thèse que la résorption accélérée après administration d'insuline s'expliquait par un passage gastrique accéléré a été prouvée. Si le glucose et le fructose étaient perfusés directement par l'intestin en évitant l'estomac, il n'y avait pas de différence entre les ani- maux traités par l'insuline et les contrôles. Ces résultats sont en contradiction avec l'hypothèse qu'un niveau bas du glucose sanguin, en augmentant la différence de concentration entre l'intestin et le sang amène une résorption plus rapide du glucose. Après administration d'insuline par voie intraveineuse, il y avait même une inhibition statistiquement significative de la résorption du glucose 10 minutes après le début de la perfusion de l'intestin grêle. Il est cependant vraisemblable que cette inhibition de la résorption est due à l'état hypo. glycémique des animaux. Un traitement préalable avec de l'insuline pendant une semaine n'amenait pas d'accélération de la résorption du glucose.

Zusammenfassung. An insgesamt 282 Ratten wurde der Einfluß von Insulin bzw. der durch Insulininjektionen hervorgerufenen Hypoglykämie auf die Resorption von Glucose- und Fructoselösungen geprüft. Nach Verabreichung dieser Monosaccharide mit der Schlundsonde konnte unter Insulineinwirkung eine schnellere Resorption der Glucose, nicht aber der Fructose festgestellt werden. Es fiel jedoch auf, daß diese raschere Resorption der Glucose an ein größeres Zuckerangebot an den Darm pro Zeiteinheit gebunden war. Die Vermutung, daß demnach die unter Insulingaben gesteigerte Glucoseresorption durch eine beschlounigte Magenentleerung zustandekommt, ließ sich mit Hilfe weiterer Untersuchungen bestätigen. Wenn nämlich Glucose und auch Fructose unter Umgehung des Magens durch den Darm perfundiert werden, ergaben sich keine Unterschiede mehr zwischen den mit Insulin behandelten Ratten und den Kontrolltieren. Vermutungen, wonach ein niedriger Blutzuckerspiegel gleichsam das Gefälle vom Darm zum Blut verstärkt und somit eine schnellere Glucoseresorption bewirkt, sind nach diesen Befunden nicht mehr haltbar. Nach intravenöser Insulinapplikation 10 Minuten vor Beginn der Dünndarmperfusion kam es zu einer statistisch signifikanten Hemmung der Glucoseresorption. Es ist jedoch anzunehmen, daß die Ursache dieser Resorptionshemmung im Schockzustand der Tiere zu suchen ist. Auch eine einwöchige Vorbehandlung mit Insulin führte nicht zu einer Steigerung der Glucoseresorption.

Key-words: Intestinal absorption, glucose fructose absorption, insulin, hypoglycemia and glucose absorption.
Die Wirkung von Insulin auf die Zuckerresorption im Dünndarm war mehrfach Gegenstand von Untersuchungen gewesen $[22,41,42,40,29,8,4,18,2]$. In den meisten Fällen war eine Steigerung der Resorption beobachtet worden. Dies steht in Einklang mit der Hypothese über den Wirkungsmechanismus des Insulins, der auf einer Steigerung der Zellpermeabilität vor allem für Glucose, jedoch auch für andere Substanzen beruhen soll $[23,24,25,31]$. Im Widerspruch zu diesen Befunden war aber mehrfach festgestellt

* Mit Unterstützung der Deutschen Forschungsgemeinschaft. worden, daß auch Insulinmangel - bei experimentell erzeugtem Diabetes mellitus - zu einer Steigerung der intestinalen Resorption führt [22, 42, 40,34, 11].

Die Ergebnisse über die Wirkung von Insulinüberschuß und Insulinmangel auf die Resorption sind allerdings nicht ohne weiteres vergleichbar, da gänzlich unterschiedliche Methoden zur Anwendung kamen. Zudem kann bei Prüfungen am lebenden Tier mit Hilfe einer Schlundsondenmethode der Mechanismus der Magenentleerung entscheidenden Einfluß auf die Resorptionsergebnisse ausüben, da es bekannt ist, daß Insulin die Magenmotilität steigert [35, 36, 16, 3]. 
Aufgabe der vorliegenden Arbeit soll sein, die Wirkung von Insulin auf die Glucose- und Fructoseresorption im Experiment am lebenden Tier mit verschiedenen Methoden zu prüfen, um den Einfuß des Hormons auf die Magenentleerung von einem echten Effekt auf die Resorption abzugrenzen.

\section{Material und Methode}

Die Versuche wurden mit männlichen Albinoratten durchgeführt (Gewicht $170-230 \mathrm{~g}$ ). Die Tiere erhielten 24 Stunden vor Versuchsbeginn keine Nahrung mehr (Ausnahmen s.u.) und wurden in Einzelkäfige mit einer Maschenweite von $1 \mathrm{~cm}$ zur Verhinderung der Koprophagie gesetzt. Die durch tägliche Insulininjektionen vorbehandelten Tiere konnten ohne Gefahr eines hypoglykämischen Schocks jedoch nur 2 Stunden hungern. Bei diesen Ratten wurde der Darm vor der Perfusion mit aqua dest. durchspült, um Kot und Nahrungsreste herauszuwaschen.

Insulin wurde bei denjenigen Experimenten, bei denen es vor Versuchsbeginn injiziert wurde, in einer schnell wirksamen Form gegeben (Actrapid ,Novo"). Bei den Langzeitversuchen, d.h. bei den Versuchen mit längerdauernder Insulinvorbehandlung wurde ein Depot-Insulin (,Horm", Hormon-Chemie) subcutan injiziert.

Bei den mit der Schlundsondenmethode durchgeführten Versuchen wurde das Insulin subcutan in der Weise verabreicht, daß 75 Minuten Abstand zwischen Insulingabe und der Mitte der jeweiligen Prüfperiode bestand. Für die Darmperfusionsversuche wurde das Insulin 30 Minuten vor Versuchsbeginn subcutan oder 10 Minuten vor Versuchsbeginn intravenös (mittels Venenkatheder in die $\nabla$. jugularis) injiziert. Bei den Langzeitversuchen erhielten die Tiere 7 Tage lang täglich morgens $20 \mathrm{E}$ Depot-Insulin subcutan. Die letzte Injektion erfolgte 24 Stunden vor Versuchsbeginn.

Schlundsondenmethode [30]. In einem ganz leichten Ätherrausch wurde den Ratten zu Versuchsbeginn $1.3 \mathrm{ml}$ Versuchslösung mit der Schlundsonde in den Magen instilliert. Die Lösungen enthielten 10\%iges Kollidon (Polyvinylpyrrolidon, durchschnittliches Molekulargewicht 30000 ), ferner $50 \%$ Glucose. 15, 30 oder 60 Minuten nach Versuchsbeginn wurden die Tiere getötet und das Abdomen eröffnet. Der Magendarmtrakt wurde nach Ligatur am Pylorus, an der Cardia und am Übergang in das Coecum herausgelöst, in Eiswasser abgekühlt und gewaschen. Dann wurde der verbliebene Magen- bzw. Darminhalt mit eisgekühltem aqua dest. herausgewaschen.

Darmperfusionsmethode $[12,13]$. Die Ratten wurden in Urethannarkose laparatomiert; dann wurden
Sonden in das Duodenum und in den unteren Teil des Dünndarms, direkt vor dem Úbergang zum Coecum eingelegt und fest eingebunden. Anschließend wurde das Abdomen verschlossen und die Perfusionslösung konnte nach vorhergehender Erwärmung auf Körpertemperatur in einem Wasserbad unter Umgehung des Magens kontinuierlich durch den Dünndarm perfundiert werden. Diese Methode erlaubt eine direkte Messung der Resorptionskapazität des Dünndarms der lebenden Ratte. Durch Verwendung von $60 \mathrm{ml}$ Prüflösung wird bewirkt, daß die Konzentration der Prüfsubstanz im Darmlumen während der Prüfzeit sich nur wenig ändert.

Chemische Nachweise. Kollidon wurde mit der von uns modifizierten Methode nach SCHUBERT [30, 39] bestimmt. Die Glucose wurde nach dem von KeILLIN und HARTREe [20] angegebenen Nachweis in der Vorschrift von HUGGET und NIXON [19] enzymatisch mit Glucoseoxydase-Peroxydase nachgewiesen (Boehringer Testverfahren). Der Nachweis der Fructose erfolgte nach RoE [37].

Durch die zusätzliche Verabreichung von Kollidon, einer nicht resorbierbaren, hochmolekularen Verbindung lassen sich die Wasserveränderungen während der Resorptionsprüfung nachweisen. Da diese Substanz nicht resorbiert wird, ist ihre Konzentrationsänderung ein Maß für die Wasserveränderung; es läßt sich also aus der Kollidon-Konzentrationsänderung die Wasserveränderung berechnen. Bei der Schlundsondenmethode wird es dadurch möglich, die Magenentleerung zu messen $[12,13,30]$. Die Resorption der Prüfsubstanz läßt sich bei Anwendung dieser sogenannten „Kollidon-Clearance" über die Berechnung der Wasserveränderung genauestens ermitteln.

Alle Analysen wurden jeweils als Dreifachbestimmungen durchgeführt.

\section{Ergebnisse:}

Aus Abb. 1 sind die mit der Schlundsondenmethode erzielten Ergebnisse für die Glucoseresorption unter dem Einfluß von Insulin zu ersehen. Mit dieser Me-

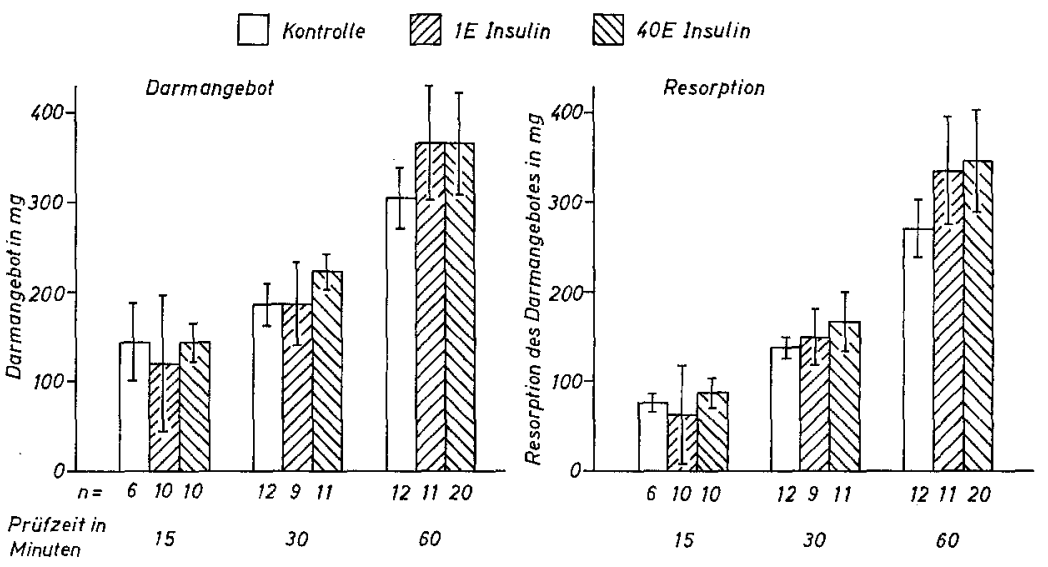

Abb. 1. Einfluß von Insulin auf das Darmangebot und die Resorption nach Verabreichung von $1.3 \mathrm{ml}$ $50 \%$ iger Glucoselösung mit der Schlundsonde 
thode wird nicht nur die Resorption im Dünndarm, sondern auch die Entleerungsfunktion des Magens geprüft. Bei Aufarbeitung des in Segmente aufgeteilten Dünndarms kann man feststellen, daß bei schnell resorbierbaren Substanzen keine Prüfsubstanz in den unteren Darmabschnitten nachweisbar ist [30]. Andererseits entspricht die orale Applikation jedoch dem physiologischen Weg der Nabrungsaufnahme und die Ergebnisse erlauben daher Aussagen über das Verhalten unter physiologischen Bedingungen. Den Ergebnissen ist $\mathrm{zu}$ entnehmen (Abb. 1, rechts), daß insgesamt nur eine schwache Steigerung der Glucoseresorption unter Einfluß von Insulin zu beobachten ist. Nach 15 Minuten sind praktisch keine Unterschiede vorhanden. Nach 30 Minuten erkennt man eine geringe Steigerung der Glucoseresorption bei Verabreichung von $1 \mathrm{E}$ Insulin, eine etwas größere Zunahme der Resorption nach $40 \mathrm{E}$ Insulin. Diese Zunahme beträgt bei $1 \mathrm{E}$ Insulin nach 30 Minuten $10.8 \mathrm{mg}(7 \%)$ und bei $40 \mathrm{E}$ Insulin $30.3 \mathrm{mg}$ Glucose $(20 \%)$. Nur die Zunahme nach $40 \mathrm{E}$ Insulin ist statistisch signifikant $(p<0.05)$. Der Untersuchungszeitraum von einer Stunde zeigt einen weiteren Anstieg der Resorption unter Insulineinfluß. $1 \mathrm{E}$ Insulin bewirkt in diesem Zeitraum eine Zunahme der Resorption von $63 \mathrm{mg}(23 \%)$, nach $40 \mathrm{E}$ Insulin beträgt die Steigerung $74.5 \mathrm{mg}(28 \%)$ gegenüber den Vergleichstieren. Beide Resorptionsänderungen sind statistisch signifikant $(p<0.05)$.

Betrachtet man die Veränderungen der Magen. entleerung (= Darmangebot) durch Insulin (Abb. 1, links), so zeigt sich, daß die Resorptionszunahme von einer gleichsinnigen Veränderung der Magenentleerung begleitet wird, d.h. die Ursache für die Resorptionssteigerung ist zumindest $\mathrm{z} . \mathrm{T}$. in einer Erhöhung der Magenentleerung zu sehen. Die prozentuale Resorption von Glucose bleibt weitgehend unverändert.

So erhöht sich die Magenentleerung nach $40 \mathrm{E}$ Insulin bei der Prüfzeit 30 Minuten von $186.7 \mathrm{mg}$ Glucose auf $227.5 \mathrm{mg}$, die Resorption bleibt aber $74 \%$. Da mit der Schlundsondenmethode, wie oben kurz ausgeführt, in erster Linie die Magenentleerung gemessen wird, geben diese Ergebnisse einen Hinweis darauf, daß die Resorption von Glucose durch Insulin wenig verändert wird. Demgegenüber hatten wir bei früheren Untersuchungen an alloxandiabetischen Tieren gefunden, daß bei der Schlundsondenmethode nicht nur die Magenentleerung, sondern auch die prozentuale Resorption gesteigert ist. Mit der Perfusionsmethode ließ sich dann die Resorptionssteigerung bestätigen [11].

Es ist augenfällig, daß die Steigerung der Magenentleerung bei Verwendung von $40 \mathrm{E}$ Insulin zeitlich früher einsetzt als bei $1 \mathrm{E}$ Insulin (Abb. 1). Wenn man mit der gleichen Methode die Fructoseresorption untersucht (Abb. 2), läßt sich die Steigerung der Magenentleerung nach 30 Minuten nur nach $1 \mathrm{E}$ Insulin feststellen (Steigerung um 150\%). Nach $40 \mathrm{E}$ Insulin ist die Magenentleerung gegenüber den Vergleichstieren nur noch um 20\% erhöht (Abb. 2, links). Nach 15 Minuten wird durch beide Dosierungen die Magenent-
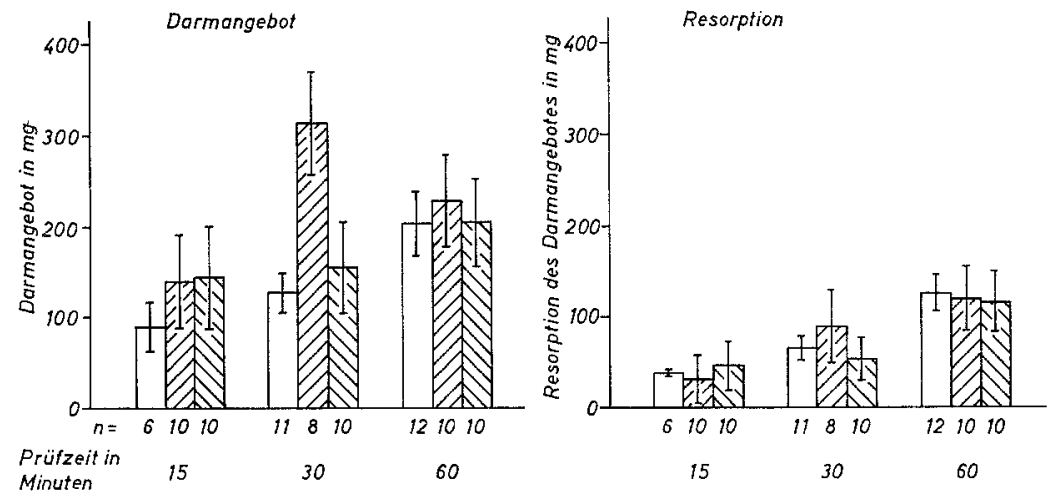

auf das Darmangebot und die Resorption nach
$30 \%$ iger Fructelosösung mit der Schlundsonde

leerung um etwa $60 \%$ erhöht. Die Fructose bewirkt bei oraler Verabreichung keine unmittelbare Erhöhung des Blutglucosespiegels und wird überdies langsamer als Glucose resorbiert. Die Tiere werden daher nicht wie bei den Untersuchungen mit Glucose aus dem hypoglykämischen Schock herausgehalten. Bei Erniedrigung des Blutglucosespiegels (durch Insulin) wird offensichtlich zuerst die Magenentleerung gesteigert. Im Schockzustand - die Tiere, die Fructose und Insulin erhalten hatten, waren nach 1 Stunde (105 Minuten nach Insulininjektion) bewußtlos - wird dann die Magenentleerung wieder gehemmt.

Eine Veränderung der Fructoseresorption war nach Insulinapplikation mit der Schlundsondenmethode nicht nachweisbar (Abb. 2, rechts).

Bei Verwendung der Schlundsondenmethode ließ sich also eine Steigerung der Magenentleerung durch Insulin nachweisen, während wir aus der unveränderten prozentualen Resorption die Schlußfolgerung zogen, daß die Resorption durch Insulin wenig beeinflußt wird. Zur Erhärtung dieser Feststellung führten wir Resorptionsuntersuchungen mit der Darmperfusionsmethode durch. Diese Methode hat den Vorteil, daß der Mechanismus der Magenentleerung ausgeschaltet wird und die gesamte Resorptionskapazität des Dünndarms ausgenützt werden kann. Durch Verabreichung von $60 \mathrm{ml}$ Flüssigkeit ist gewährleistet, daß die Glucosekonzentration im Darmlumen während des Untersuchungzeitraumes konstant gehalten wird. 30 Minuten vor Versuchsbeginn wurden 40 E Alt-Insulin (Actrapid „Novo“) subcutan injiziert. Die Untersuchungsergebnisse zeigen, daß die Resorption von Glucose oder Fructose durch einmalige Injektion einer hohen Dosis Insulin nicht gesteigert werden kann 
(Abb. 3). Die geringen Unterschiede sind statistisch nicht signifikant $(p>0.05)$.

Auch einwöchige Vorbehandlung der Tiere mit täglich $20 \mathrm{E}$ Insulin führte nicht zu einer gesteigerten,

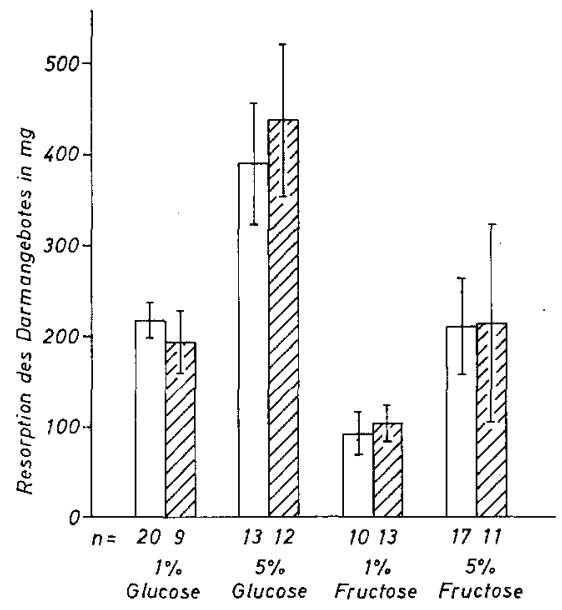

Abb. 3. Die Resorption 1\%iger und 5\%iger Glucose- bzw. Fructoselösungen aus dem perfundierten Dünndarm der Ratte 30 Minuten nach subeutaner Injektion von 40E Insulin Actrapid. (Perfusionslösung: $60 \mathrm{ml}$, Versuchsdauer: 60 Minuten)

sondern sogar zu einer herabgesetzten Glucoseresorption (siehe Abb. 4). Bei dieser Untersuchungsreihe konnte den Tieren die Nahrung nicht für 24 Stunden entzogen werden, da sie sonst im hypoglykämischen Schock verstorben wären. Eine längere Hungerperiode als 2 Stunden wirkte sich bereits auf die Resorption aus. Bei 2 Stunden hungernden Tieren, die 24 Stunden vor Versuchsbeginn die letzte Insulininjektion erhalten hatten, war die Glucoseresorption nur um $6.5 \%$ vermindert $(p>0.05)$. Ließen wir die Tiere dagegen 5 Stunden hungern, so befanden sie sich bereits im Schockzustand. und die Glucoseresorption war um 48\% herabgesetzt. Bei einer dritten Gruppe von Tieren wurden zusätzlich zur einwöchigen Vorbehandlung noch 40 E Alt-Insulin 2 Stunden vor Versuchsbeginn subcutan injiziert und gleichzeitig die Nahrung entzogen. Diese Tiere befanden sich ebenfalls zu Beginn des Versuchs im hypoglykämischen Schock; ihre Resorption war um $22 \%$ geringer 'als die der Vergleichstiere. Bei zwei Kontrollreihen, bei denen das Intervall des Nahrungsentzugs variiert wurde, die Tiere aber kein Insulin erhielten, erwies sich, daß die Glucoseresorption nur vom Zeitpunkt der Insulinapplikation und damit vom Ausmaß des hypoglykämischen Schocks abhängt (siehe Abb. 4).

In der nächsten Untersuchungsreihe wurde der Einfluß des kurz vor Versuchsbeginn intravenös injizierten Insulins geprüft. Aus Abb. 5 und Tab. 1 ist zu ersehen, daß mit steigender Insulinkonzentration die Glucoseresorption abnimmt. Gleichzeitig mit der Einschränkung der Glucoseresorption ist aber auch die Wasserresorption vermindert (siehe Tab. 1). Injektion

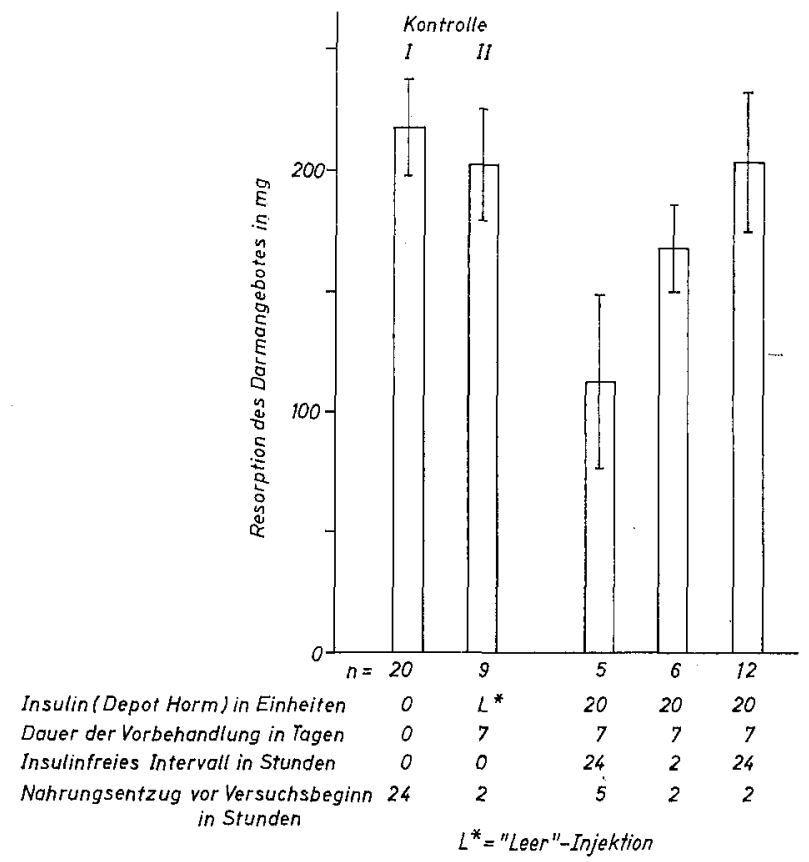

Abb. 4. Einfluß der Vorbehandlung mit Insulin auf die Resorption 1\%iger Glucoselösungen aus dem perfundierten Dünndarm der Ratte. (Perfusionslösung: $60 \mathrm{ml}$, Versuchsdauer: 60 Minuten)

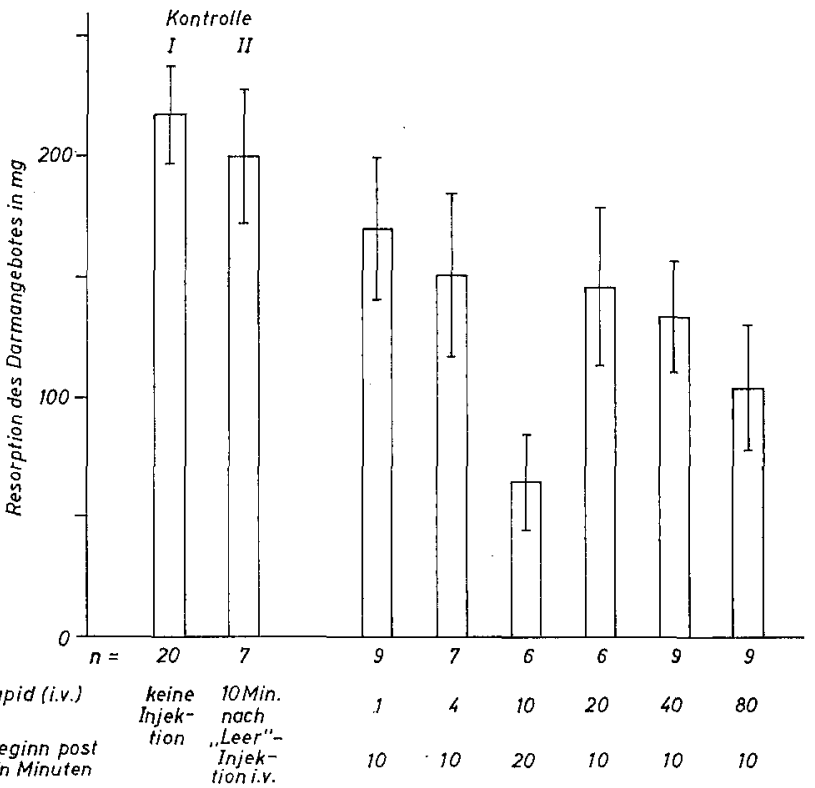

Abb. 5. Einffuß intravenöser Verabreichung von Insulin auf die Resorption $1 \%$ iger Glucoselösungen aus dem perfundierten Dünndarm der Ratte. (Perfusionslösung: $60 \mathrm{ml}$, Versuchsdauer: 60 Minuten)

des insulinfreien Lösungsmittels (,Leer"-Injektion) hatte keinen Einfluß auf die Resorption. Intravenöse Injektion von $80 \mathrm{E}$ Aatrapid 10 Minuten vor Versuchsbeginn führte zu einer Hemmung der Glucoseresorption 
um $52 \%$, während die Wasserresorption um $64 \%$ vermindert war. Injektion von $4 \mathrm{E}$ Insulin hemmte die Glucoseresorption um 30\% und die Wasserresorption um $28 \%$. Bei $1 \mathrm{E}$ Insulin ergaben sich Hemmungen um $20 \%$ bzw. um 37\%. Wurde dagegen der Zeitraum zwischen Injektion und Perfusionsbeginn auf $20 \mathrm{Minu}$ ten ausgedehnt, so führten $10 \mathrm{E}$ Insulin zu einer Hemmung der Glucoseresorption um 70\% und zu einer Hemmung der Wasserresorption um 68.5\%. - Wir schließen aus diesen Ergebnissen, daß die Resorptionshemmung ein sekundärer Vorgang ist, der z.B. durch einen Einfluß auf die aktive Resorption aus dem Dünndarm ausüben können. Die Dünndarmresorption von Glucose bei weiblichen Versuchstieren ist um 20\% höher als die von gleichaltrigen männlichen Tieren [10]. Ovarektomie führt dagegen zu einer 25\% igen Minderung der Glucoseresorption, also zu einer Angleichung an die Resorptionsverhältnisse bei männlichen Tieren [1]. Thyroxin steigert und Cortison hemmt die Glucoseresorption $[40,1,17,6]$. Bei alloxandiabetischen Versuchstieren wurde mehrfach eine Steigerung der Glucoseresorption $[40,2,34,11]$ festgestellt. Anderer-

Tabelle 1. Einfluß intravenöser Verabreichung von Insulin auf die Resorption $1 \%$ iger Glucoselösungen aus dem perfundierten Dünndarm der Ratte

\begin{tabular}{|c|c|c|c|c|c|c|c|c|}
\hline \multirow{3}{*}{$\begin{array}{l}\text { Insulinvor- } \\
\text { behandlung } \\
\text { in } \mathrm{E}\end{array}$} & \multirow{3}{*}{$n$} & \multicolumn{2}{|c|}{ Perfusionslösung: $60 \mathrm{ml}$} & \multicolumn{4}{|c|}{ Versuchsdauer : 60 Minuten } & \multirow{3}{*}{$\begin{array}{l}\text { Wiedererhaltene Elüssig } \\
\text { keitsmengen in \% des } \\
\text { Ausgangswertes }\end{array}$} \\
\hline & & \multirow{2}{*}{$\begin{array}{l}\text { Perfusionsbeginn } \\
\text { post injectionem } \\
\text { in Minuten }\end{array}$} & \multirow[t]{2}{*}{$\begin{array}{l}\text { Resorption } \\
\text { in mg }\end{array}$} & \multirow[t]{2}{*}{$s x(\mathrm{mg})$} & \multirow[t]{2}{*}{$\begin{array}{l}\text { Resorption } \\
\text { in \% }\end{array}$} & \multicolumn{2}{|c|}{$\begin{array}{l}\text { Blutglucose-Konzen- } \\
\text { tration }\end{array}$} & \\
\hline & & & & & & $\begin{array}{l}\text { Beginn } \\
\mathrm{mg} \%\end{array}$ & $\begin{array}{l}\text { Ende } \\
\mathrm{mg} \%\end{array}$ & \\
\hline 1 & 9 & 10 & 169.9 & 13.56 & 28.3 & 94 & 109 & 76.4 \\
\hline 4 & 7 & 10 & 151.02 & 15.10 & 25.2 & 69 & 104 & 72.5 \\
\hline 10 & 6 & 20 & 65.4 & 8.70 & 10.9 & -1 & - & 88.0 \\
\hline 20 & 6 & 10 & 154.98 & 15.24 & 24.33 & -1 & 99 & 75.5 \\
\hline 40 & 9 & 10 & 133.26 & 10.98 & 22.21 & 40 & 63 & 79.4 \\
\hline 80 & 9 & 10 & 104.1 & 12.42 & 17.35 & 54 & 79 & 86.3 \\
\hline $\begin{array}{l}\text {,Leer"4- } \\
\text { Injektion }\end{array}$ & 7 & 10 & 200.28 & 12.12 & 33.38 & 97 & 218 & 68.3 \\
\hline $\begin{array}{l}\text { keine } \\
\text { Injektion }\end{array}$ & 20 & - & 217.71 & 9.75 & 36.285 & - & - & 62.1 \\
\hline
\end{tabular}

${ }_{1}^{1}$ Blutglucosebestimmungen konnten nicht durchgeführt werden, da sich die Tiere im Schock befanden.

$22.0 \mathrm{ml}$ Insulin-freies Lösungsmittel.

eine verminderte Durchblutung des Dünndarms in der Hypoglykämie bewirkt sein könnte. Für diese Ansicht spricht, daß die Wasserresorption, die im allgemeinen als passiver Vorgang angesehen wird, im gleichen Sinn wie die Glucoseresorption beeinflußt wird.

\section{Diskussion}

Glucose und auch andere Monosaccharide werden nach den z.Z. gültigen Theorien durch einen aktiven Transport mit Hilfe eines Carriers durch die Zellmembran in die verschiedenen Zellen eingeschleust $[18,7$, $23,24,25,44,38,32,33,21,5]$.Auch für die selektive Resorption im Dünndarm wird das Vorhandensein von Transportvorgängen gefordert [7, 44]. Dadurch werden die Unterschiede in der Resorptionsgeschwindigkeit der einzelnen Monosaccharide erklärt. Glucose und Galaktose z.B. werden aktiv transportiert, während die wesentlich langsamer resorbierbare Fructose wahrscheinlich und der Zuckeralkohol Sorbit mit Sicherheit nur durch passive Diffusion aus dem Dünndarm in das Blut übertreten können. Neuere eigene Untersuchungsergebnisse lassen allerdings auch Zweifel an der Bedeutung der überwiegend aktiven Resorption für Glucose und Galaktose - zumindest bei höheren Konzentrationen aufkommen $[13,14,15]$. Es ist bekannt, da $\beta$ auch andere Hormone, wie z.B. Thyroxin $[40,1]$ und Cortison $[40,17,6]$ sowie weibliche Sexualhormone [i0] seits soll auch eine Hypoglykämie durch Erhöhung des Konzentrationsgefälles vom Darm zum Blut die Glucoseresorption steigern $[18,26,27]$.

SolaIrac fand bei Verwendung der Schlundsondenmethode eine Steigerung der Glucoseresorption in der Hypoglykämie [40]. Er führte bei Ratten eine fünftägige Vorbehandlung mit Insulin durch, wobei er täglich $40 \mathrm{E}$ Insulin verabreichte. Die mit Insulin vorbehandelten Tiere zeigten eine Steigerung der Glucoseresorption auf das Zweieinhalbfache der Vergleichstiere. Bei der von SolaIRac verwendeten Schlundsondenmethode ist jedoch der Einwand zu erheben, daß die Wirkung des Insulins auf die Magenmotilität nicht berücksichtigt wurde (vergleiche eigene Ergebnisse). Von Mackay und Clark [28] wurde eine erhöhte Nahrungsaufnahme nach Insulinverabreichung festgestelllt. Sie führten diese Insulinwirkung nicht nur auf den gesteigerten Nahrungstrieb, sondern auch auf eine beschleunigte Magenentleerung zurück. Von QUIGLeY und Mitarbeitern [35, 36] war nämlich bereits früher eine Steigerung der Magenmotilität nach Insulin beschrieben worden. Dieser Befund wurde neuerdings von AYLETT [3] bestätigt und kann auch durch unsere Untersuchungen als belegt gelten. Wie wir zeigen konnten, wird die nach Insulinapplikation bei der Schlundsondenmethode beobachtete Resorptionssteigerung allein durch eine Beschleunigung 
der Magenentleerung und damit durch ein größeres Angebot resorbierbarer Substanzen an den Darm pro Zeiteinheit bewirkt (Abb. 1).

Die in vitro-Untersuchungen verschiedener Autoren führten zu völlig unterschiedlichen Ergebnissen. Auf die vermehrte Galaktoseaufnahme - Galaktose wird über den gleichen Mechanismus wie Glucose resorbiert - durch den überlebenden Darm alloxandiabetischer Ratten hatte Insulin im Inkubationsmedium nur einen geringen Einfluß, während intravenöse Insulingaben vor der Tötung der Tiere wirkungslos blieben [7]. Mit der gleichen Methode gelang es jedoch neuerdings AuLSEBRook [2] nachzuweisen, daß sich die Glucoseresorption des überlebenden Darmes durch Insulinvorbehandlung der Tiere steigern läßt. Bei alloxandiabetischen Ratten war die Glucoseresorption ebenfalls gesteigert. Zusätzliche Insulinvorbehandlung war bei diesen Tieren ohne Wirkung.

Sols und Larralde [42] konnten die Glucoseresorption von alloxandiabetischen Tieren in vivo durch Verabreichung von $2 \mathrm{E}$ Insulin pro $100 \mathrm{~g}$ Körpergewicht um weitere $8 \%$ steigern. Die Autoren führen die Wirkung des Insulins auf die Resorption bei alloxandiabetischen Tieren auf das Absinken des Blutglucosespiegels zurück. Dagegen hatten LASZT und VogEL [22] mit einer ähnlichen Versuchsmethode eine Hemmung der Glucoseresorption durch Insulin bei alloxandiabetischen Tieren festgestellt. Wiederum ein anderes Resultat ergab sich bei den Untersuchungen von HoRvatH und WIX, die - ebenfalls in vivo - eine von der Höhe der Hypoglykämie abhängige Resorptionssteigerung erhielten [18]. Wenn die Hypoglykämie durch gleichzeitige Glucoseinjektion kupiert wurde, blieb die Resorptionssteigerung aus.

Manome und KuRIaki [29] führten eine Perfusion des Darms der lebenden Ratte mit einer Methode durch, die der von uns verwendeten ähnlich ist. Das Insulin wurde ebenfalls intravenös verabreicht. Dabei wurde festgestellt, daß $0.5 \mathrm{E} / \mathrm{kg}$ Körpergewicht wirkungslos waren. Injektion von $1.5 \mathrm{E} / \mathrm{kg}$ Körpergewicht führten zu einer Steigerung der Glucoseresorption um $25 \%$ und $5 \mathrm{E} / \mathrm{kg}$ Körpergewicht $\mathrm{zu}$ einer Steigerung um $55 \%$ gegenüber den Kontrolltieren und auch gegenüber jenen Tieren, die nur $0.5 \mathrm{E} / \mathrm{kg}$ Körpergewicht erhalten hatten.

Wir konnten keine Bedingungen finden, unter denen eine Steigerung der Glucoseresorption durch Insulin erzielt werden konnte. Weder eine einwöchige Vorbehandlung, noch eine subcutane oder intravenöse Injektion von Insulin führten zu einer gesteigerten Glucoseresorption. Im Gegenteil, je höher die Insulindosierung war bzw. je tiefer sich die Tiere im Schockzustand befanden, umso größer war die Hemmung der Glucoseresorption. Jedoch lassen die am Ende der Perfusionsperiode vorhandenen Blutglucosespiegel von über $50 \mathrm{mg} \%$ (Tab. 1) offen, ob der durch die hohe intravenöse Dosierung bewirkte Schock allein durch eine Hypoglykämie verursacht wurde. Leider waren keine
Verlaufskontrollen zu erhalten, da auf Grund des Schockzustandes kein Blut vom Schwanz der Tiere genommen werden konnte. Andererseits war auch nicht die Injektion der Flüssigkeit die Ursache für die Resorptionshemmung; denn bei Injektion der gleichen Menge Lösungsmittel war die Glucoseresorption praktisch nicht vermindert (Abb. 5 und Tab. 1). Manome und KURIAKI [29] hatten bei ihrer höchsten Dosierung ( $5 \mathrm{E} / \mathrm{kg}$ Körpergewicht) etwas mehr als $1 \mathrm{E}$ Insulin pro Ratte injiziert und dabei eine Glucoseresorptionsteigerung um 55\% erzielt. Dieser Befund konnte durch unsere Versuche nicht bestätigt werden. Auch $1 \mathrm{E}$ Insulin in vivo bewirkte eine deutliche Hemmung der Glucoseresorption. Genausowenig können wir die Befunde von HoRvatH und WIX [18] erklären. Es ist nicht zu erwarten, daß die Höhe des Blutglucosespiegels einen Einfluß auf die Glucoseresorption ausübt, da bei Perfusion von 1\% Glucose immer ein Glucosekonzentrationsgefälle vom Darm zum Blut besteht (die Blutglucosespiegel am Ende der Perfusion ohne Insulininjektion betragen nämlich etwa $0.2 \%$ ). Daneben handelt es sich beider Glucoseresorption um einen, ,aktiven “ Vorgang, der auch bergauf erfolgt. Dabei ist es gleichgültig, worin der Mechanismus dieses Transportes besteht. Die einzige Erklärungsmöglichkeit für die unterschiedlichen Ergebnisse der anderen Autoren sehen wir darin, daß keine „Darm-Clearance-Methode“ (siehe Methodik) durchgeführt wurde und dadurch die Resorption nur ungenau erfa Bt, werden konnte. Wenn man nämlich die von uns miterfaßten, mit der Glucoseresorption gleichsinnig verlaufenden Veränderungen der Wasserresorption methodisch nicht berücksichtigen kann, müssen zwangsläufig falsche Schlußfolgerungen gezogen werden.

Eine gewisse Bestätigung unserer Ergebnisse gibt die erst kürzlich erschienene Arbeit von BEYREISs, MüLLER und Strack [4], die eine anhaltende Hemmung der Galaktoseresorption durch Insulin beobachten konnten. Diese Autoren führten beim Kaninchen über einen im Duodenum liegenden Katheter eine Dauerinfusion von Galaktose in den Dünndarm durch. Bei zusätzlicher intravenöser Infusion von $0.2 \mathrm{E}$ Insulin pro kg Körpergewicht und. Stunde kam es zu einem deutlichen Absinken des Blutgalaktosespiegels. Nach Tötung der Tiere konnte im Dünndarm der mit Insulin behandelten Tiere wesentlich mehr Galaktose nachgewiesen werden als bei den Vergleichstieren. Es wurde auf eine spezifische Wirkung des Hormons auf die aktive Resorption der Monosaccharide geschlossen. Leider kann bei der von den Autoren angewandten Methode ebenfalls keine Aussage über die Wasserresorption gemacht werden.

\section{Literatur}

[1] Arrhausex, T.L. and M. Stockholm: Influence of the thyroid gland on absorption in the digestive tract. Amer. J. Physiol. 123, 577 (1938).

[2] AULsEbrook, K.A.: Intestinal transport of glucose and sodium: Changes in alloxan diabetes and effects of insulin. Experientia 21, 346 (1965). 
[3] Aycert, P.: Gastric emptying and change of blood glucose level as effected by glucagon and insulin. Clin. Sci. 22, 171 (1962).

[4] Beyretss, K., F. MülLer und E. Strack : Über die Resorption von Monosacchariden. Z. ges. exp. Med. 138, 227 (1964).

[5] Blemer, N.M., and R.B. Fisher: The action of insulin on the isolated rat heart. J. Physiol. 123, 260 (1954).

[6] CAPELII, V., and L. FACCONI: Effect of prolonged administration of cortisone and adrenalcorticoid function studied by intestinal glucose absorption. Boll. Soc. ital. Sper, 32, 1046 (1956).

[7] Crane, R.K.: Intestinal absorption of sugars. Physiol. Rev. 40, 789 (1961)

[8] - Effect of alloxan-diabetes on the active transport of sugars by rat small intestine in vitro. Biochem. Biophys. Res. Com. 4, 436 (1961).

[9] DAVIs, A., and F. Brooks: Gastric secretion continuously recorded blood sugar, and plasma steroids after insulin. Amer. J. Physiol. 204, 142 (1963).

[10] Frsher, R.B., and D.S. PArsons: Galactose absorption from surviving small intestine of the rat. J. Phy siol. 119, 224 (1953).

[11] Förster, H., und H. Mehrert: Untersuchungen zur Beeinflussung der Glucose- und Fructoseresorption durch Insulin, Cortison und Thyroxin sowie Alloxandiabetes. Dtsch. Ges. Verd. Stoffw. krkh. 22. Tag., Gastroenterologia 104, Suppl. 166 (1965).

[12] - - Resorptions- und Sekretionsprüfungen bei Perfusion des Rattendarms mit hypo- und hypertonischen Glucoselösungen. Klin. Wschr. 41, 1167 (1963).

[13] - Experimentelle Untersuchungen zur aktiven Resorption von Zuckern am lebenden Tier. I. Mitt. Zur Frage einer Substratsättigungskurve für die Resorption von Glucose und Galaktose. Klin. Wschr. 43, 834 (1965).

[14] - , W. Katser und H. Mehnert: Tierexperimentelle Untersuchungen zur aktiven Resorption. II. Mitt. Prüfung der Glucoseresorption gegen einen erhöhten Blutglucosespiegel. Klin. Wschr. 43, 839 (1965).

[15] - - Tierexperimentelle Untersuchungen zur aktiven Resorption. III. Mitt. Die Ausscheidung von Glucose in das Darmlumen der Ratte bei hohem Blutglucosespiegel. Klin Wschr. 43, 844 (1965).

[16] Gage, J.M., A. Ochsner and R. A. CutTing: Effect of insulin and glucose on normal and obstructed intestine. Proc. Soc. expt. Biol. 29, 264 (1932).

[17] Gyökössy, J., P. KertaI und G. Ludany: Wirkung des Cortisons und ACTH auf die Glucoseresorption aus dem Darm. Arch. int. Pharmacodyn. 101, 228 (1955)

[18] Horvath, I., and G. Wrx: Hormonal influences on glucose resorption from the intestine. Acta physiol. hung. 2, 445 (1965).

[19] Hugget, A, and D.A. Nixon: Enzymic determination of blood glucose. Biochem. J. 66, 12 P (1957).

[20] KeIlim, P., and E.F. Hartree : Properties of actalase. Catalysis of coupled oxidation of alcohols. Bio. chem. J. 39, 293 (1945).

[21] Kipars, D.M., and C. CoRI: Studies of insulin on pentose uptake by the diaphragm. J. biol. Chem. 224, $681(1957)$.

[22] LASzT, L., and H. VoGEL: Resorption of glucose from the small intestine of the alloxan-diabetic rat. Nature $\mathbf{1 5 7}, 551(1946)$.

[23] Levine, R., M. Goudstein, B. Huddiestone and S. KrEIN : Action of insulin on the permeability of cells to hexoses, as studied by its effects on the distribution of galactose. Amer. J. Physiol. 163, 70 (1950).

[24] - - Effect of Insulin on the rate of transfer of sugar across cell barriere. Brookhaven Symposia in Biology 5, 73 (1952).

[25] - - On the mechanism of action of insulin. Recent Progr. Hormone Res. 11, 343 (1955).

[26] LoRAu, M.: Importance des modifications de la glycémie dans la régulation de la vitesse d'absorption de solution de glucose de différentes concentrations chez l'animal intact. Experientia 15, 192 (1959)

[27] - L'absorption intestinale du glucose est-elle un phénomène réversible? C. R. Acad. Sci. 238, 842 (1963).

[28] MacKay, E.M., and W.G. Clark: The rate of glucose absorption from the intestine of the rat. Amer. J. Physiol. 135, 182 (1941).

[29] Manome, s.H., and K. Kuriaki : Effect of insulin, phlorizin and some metabolic inhibitors on the glucose absorption from the intestine. Arch. int. Pharma. codyn. 130, 187 (1961).

[30] Mehnert, H., and H. Förster: Die Prüfung der Resorption von Glucose, Fructose und Sorbit mit einer neuen Methodik. Klin. Wschr. 39, 596 (1961).

[31] Park, C.R., D. Reinwern, M.J. Henderson, E. CaDENAS and H.E. MORGAN: The action of insulin on the transport of glucose through cell membrane. Amer. J. Med. 26, 674 (1959).

[32] - J. Borsstern and R.L. Pork: Effect of insulin on free glucose content of rat diaphragm in vitro. Amer. J. Physiol. 182, 12 (1955).

[33] - , and L.M. JoHrson: Effect of insulin on transport of glucose and galactose into cells of rat muscle and brain. Amer. J. Physiol. 182, 17 (1955).

[34] PAULS, F., and D.R. DRURY: The rate of absorption from the intestine of diabetic rats. Amer. J. Physiol. 137, 242 (1942).

[35] QUIGLEY, J.P., and E.J. SALOMON : Action of insulin on the motility of gastrointestinal tract. Amer. J. Physiol. 191, 488 (1930)

[36] - , V. Johnson and E.J. SAtomon: Action of insulin on the motility of the gastrointestinal tract. I. Action on the stomach of normal fasting man. Amer. J. Physiol. 90, 89 (1929).

[37] Roe, J.H.: A colorimetric method for the determination of fructose in blood and urine. J. biol. Chem. 107, 15 (1934).

[38] Rosenperg, T., and W. WrLbrandt: Kinetics of membrane transport involving chemical reactions. Exp. Cell Res. 9, 49 (1955).

[39] Schubert, R., und H. WAGNER: Normalausscheidung von Periston $\mathrm{N}$ bei Anwendung eines neuen Kollidonnachweises. Z. ges. exp. Med. 131, 90 (1959).

[40] Soulatrao, A.: La régulation neuro-endocrinienne de l'absorption intestinale des glucides. Ann. En. docrinol. 8, 377 (1947).

[41] Sols, A.: Acción de la insulina sobre la absorción intestinal. Rev. esp. Fisiol. 7, 1 (1951).

[42] - , S. VIDAL und J. LARRaLde : Effect of insulin on the intestinal glucose absorption in alloxan-diabetic rats. Nature 161, 932 (1948).

[43] WILBRANDT, W., and L. LASZT: Untersuchungen über die Ursachen der selektiven Resorption der Zucker aus dem Darm. Biochem. Z. 259, 398 (1933).

[44] WILsoN, T.H.: Intestinal absorption. PhiladelphiaLondon p. 69 (1962).

Priv.-Doz. Dr. H. MehnerT

III. Med. Abteilung

des Krankenhauses München-Schwabing 8 München, Kölner Platz 1 